\title{
Author Index Vol. 58, 1997
}

Vol. 58, Supplement 1 and 2 have their own Author Indexes

Aadland,E. 176

Abel,P.W. 147

Adjalla,C. 64

Adler,G. 50,115

Alberti,A. 293

Alian,H. 494

Al Moutaery, A.R. 129

An, X. 10

Andrén-Sandberg, Å. 542

Arakawa,T. 120,232,299,311

Arastéh,K. 218

Ares,J.J. 147

Arisawa, T. 332

Arnold, R. 240

Asada,Y. 19

Asahara, M. 225

Asai, J. 332

Baartman, E.A. 415

Bachem,M. 50

Bailey, D. 155

Banci,M. 498

Barthellémy-Bialas, S. 98

Bartsch,R. 155

Bassi,C. 536,570

Basson, M.D. 34

Beckh,K. 115

Begent, R. 155

Beger,H.G. 501,529,570,578

Belleville, F. 64

Beltrandi,E. 431

Beneduce, F. 58

Benhayoun, S. 64

Benvegnú, L. 293

Ben-Yehuda, D. 494

Beste,M. 396

Bianchi,G. 379

Billi,P. 431

Birk,D. 578 


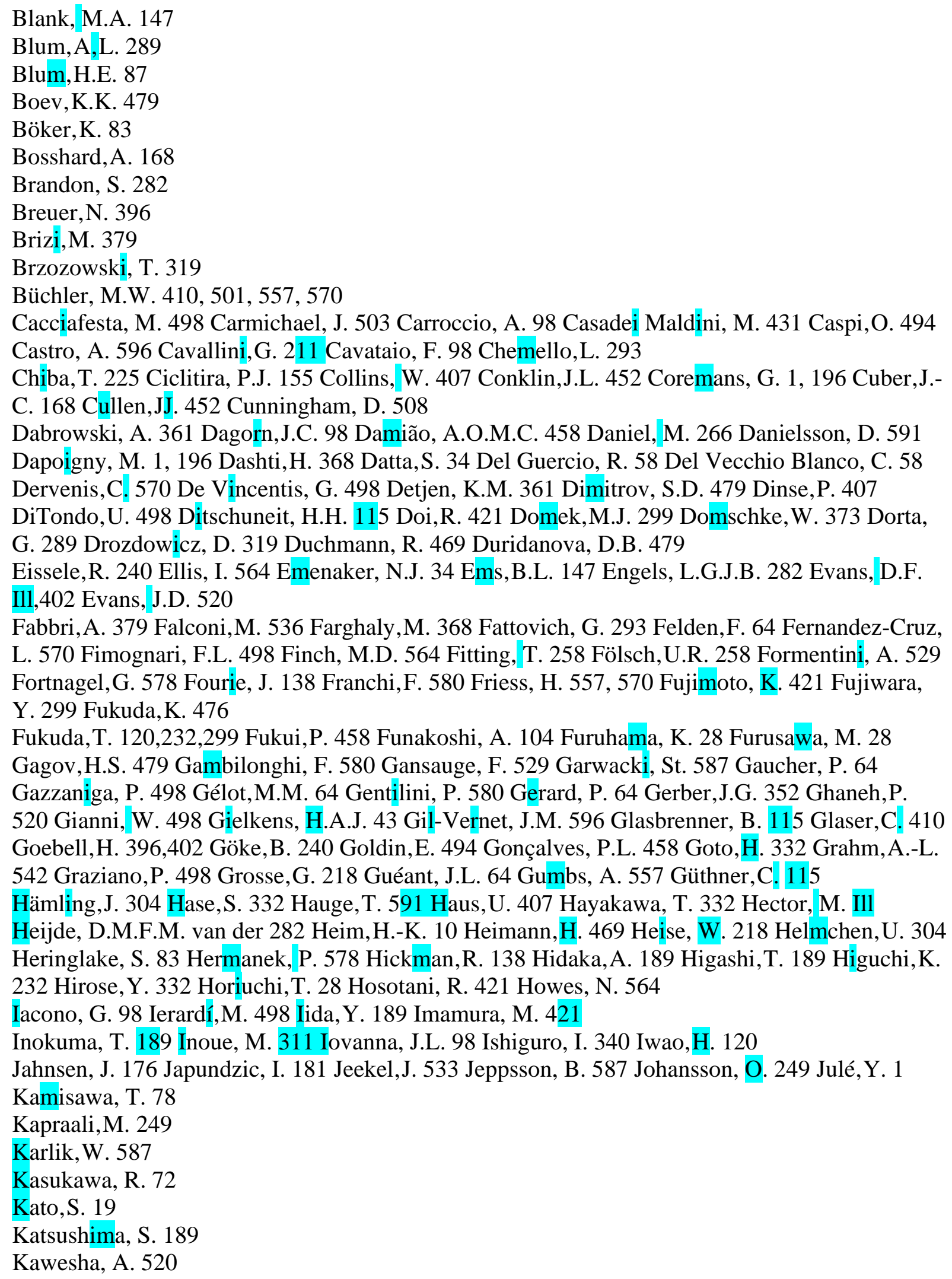


Kerr,DJ. 570

Khoursheed, M. 368

Kihara,Y. 389

Kilian,P. 10

Kim,S. 120

Kinoshita,Y. 225

Kishi,K. 225

Kiso,T. 389

Kitamura,N. 225

Kitazumi, S. 464

Klatt,S. 115

Kleeff,J. 557

Kobayashi,K. 120,232,299,311

Kobayashi,T. 340

Kodama, M. 476

Koeppen,M. 218

Kokubun, M. 72

Kondo, M. 464

Konishi,J. 189

Konturek, J.W. 373

Konturek, S.J. 319,373

Kosaric,B. 181

Koshiba,T. 421

Kuroda,M. 72

Laçaine, F. 570 L’Age,M. 218 Lafii,G. 580 Lambert, D. 64 Lamers, C.B.H.W. 43 Laudanna, A.A. 458 La Villa, G. 580 Lazzeri, C. 580 Ledlow,A. 452 Lee,J.-U. 421 Lemoine, N.R. 550 Leonardo, G. 58 Lerch,M.M. 50 LiPani,M. 98 Lieber,C.S. 203

\section{KAHGEK}

Fax+ 41613061234 E-Mail karger@karger.ch www.karger.com

(C) 1997 S.KargerAG, Basel

601

Lieverse, R.J. 43 Link, K.-H. 529,570 Logsdon, CD. 361 Loguercio, C. 58 Lohse,A.W. 469 Loser, C. 258

147

McCafferty, D.-M.

McLeod,H. 138

Maga,T. 389

596

Malagelada, J.-R.

Mall,A.S. 138

Mancini, R. 431

Manns, M. 83

Marasí,L. 98

Marchesini, G. 379

Marigliano, V. 498 
Marín,L 249

Masclee, A.A.M. 43

Masuda, M. 104

Matsushima, Y. 225

Maurer, C.A. 410

Mearin, F. 596

Merrifield, E. 138

Meslin,J.-C. 168

Meyer zum Büschenfelde, K.-H.

Mikami,Y. 389

Minamiyama, Y. 311

Mitsuyama, K. 271

Miyamoto, Y. 421

Miyasaka, K. 104

Miyata, M. 72

Miyazawa, K. 225

Mizuno, M. 389

Mohan, A.N. 266

Montalto, G. 98

Mostertz,P. 218

Mountford,R. 564

196

Müller-Lissner, S.

Murray, J.A. 452

Naito,Y. 464 Nakamura, H. 476 Neoptolemos, J.P. 501, 520, 564,

570 Netz, S. 10 Nicolas, J.P. 64 Niedobitek, F. 218 Nishida,K. 340 Nishimaki, T. 72 Niwa,Y. 332 Noventa, F. 293

Obara,K. 72 O’Brien, L.M. 147

Obst,C. 218 Ochiai, H. 72 Ohira,H. 72 Ohkusa,T. 384 Ohta,Y. 340 Oi,H. 189 Okada,H. 389

Okamura,J. 189 Okayasu, I. 384 Okon,E. 494 Or,R. 494 Osnes, M. 437 Osnes, T. 437 Overbeek, S.E. 415

Pace,F. 1,196 Pajdo, R. 319 Papachristov, E. 529 Pastoor, C.J. 282 Pawlik,T. 373 Payne, N.A. 352 Pederzoli,P. 536,570 Pedrazzoli, S. 536 Penning, C. 43 Persson, J. 591469 Pezzilli,R. 431 Pieramico, O. 115 Piller,M. 10 Plaisancié, P. 168 Popescu,R.A. 508 Printz,H. 240

Radhakrishnan, S. 266 Raedler,A. 304 Rakic-Stojiljkovic, L. lí Raknerud,N. 176 Raraty, M. 564 Renkes, P. 64 Requadt, P. 240 Reubi,J.C. 410 Rietveld,T. 415 Rijken,J. 282 Røseth,A.G. 176 Ross,P.J. 508 Rothmund,M. 240 Ruiz-Gutierrez, V. 161 Russel, M.G.V.M. 282

Sachdev,G.K. 266 Sakata, R. 444 Sandstad, O. 437 Sapsford,R. 155 Sata,M. 444 Sato,C. 384 Sato,E.F. 311

Schipper, J. 43 Schlenzka,R. 240 Schmidt,W. 218 Schönfeld, J. von Ill,396, 402 Schreiber,S. 304 Scopinaro,F. 498 Seino, Y. 476 Sewing, K.-F. 10 Shafik,A. 489 Sharma,P. 587 Shigeno,C. 189 Sipahi,A.M. 458 Skar,V. 437 Skörde,J. 218 Sliwowski, Z. 319 Smout,A. 1, 196 Söderman, C. 249 Spahn,T.W. 469 Stachura,J. 319 Stockbrügger, R.W. 1,196,282 Sugiyama, N. 28 Sulkowski,U. 407 Sutton,R. 564 Swart, GR. 415

Tabata,I. 78 Tabuchi,Y. 28 Tajima, T. 78 Takagi, T. 72 Takahashi, S. 464 Takashimizu, I. 384 Takehara, K. 19 Takeuchi,K. 19 Takeuchi,R. 189 Talamini, G 211 Tanaka,M. 120 Tanaka,S. 
389 Taniguchi, T. 476 Tanikawa,K. 271,444 Taranto,D. 58 Tariq,M. 129 Tarnawski, A. 299 Tateishi, H. 271 Theodorsson, E. 249 Thulesius, O. 368 Tojo,J. 72 Tominaga, K. 120 Tominaga, M. 476 Tomoda,J. 389 Tomoyose, M. 271 Torimura, T. 444 Tougas,G. 196 Toyonaga, A. 271 Tscherny, M. 240 Tsuji, T. 389

Tsukada, H. 476 Tsukamoto, Y. 332 Tsushima, K. 78 Turi,S. 50 Turnbull,G. 155 Ueno,T. 444 Uno,H. 232 Uribe,A. 249 Uske,A. 289

Vazquez, CM. 161 Vecchiarino, S. 580 Vezozzo, D.P. 458 Vitale,L.M. 58

Wada,M. 421 Wagner, A.C.C. 240 Wallace, J.L. 147 Wang,H.-Y. 225 Waters, J.S. 508

Wattimena, J.L.D. 415 Weichetek,M. 587 Weidenbach, H. 50 Weiss, R. 218 Weisshaar, P.S. 147 Weizsäcker, F. von 87 Whorwell, P.J. 1, 196 Wieland, S. 87 Wienbeck,M. 196 Williams, J.A. 361 Wingate,D.L. Ill,402 Wyle,F. 299

Yamaguchi, T. 464 Yamamoto, H. 464 Yasuhiro,T. 19 Yoshida,N. 464 Yoshida,Y. 78 Yoshikawa, T. 464 Yoshinaga,F. 389 Yu,H. 311

Zamboni, G. 211 Zee, R. van der 469 Zelig,O. 494 Zoli,M. 379 Zotz,R.B. 396 Zubaidi, G. 266

602

Digestion Vol. 58, 1997

Author Index 